\title{
Clinical Course of the Benign Prostate Hyplasia Patients during the Intermittent Use of 5-Alpha Reductase Inhibitors
}

\author{
Kwibok Choi, Byounghoon Kim, In-Chang Cho, Seung Ki Min
}

Department of Urology, National Police Hospital, Seoul, Korea

Purpose: 5-Alpha reductase inhibitors (5ARI), inhibit the conversion of testosterone to dihydrotestosterone, which is essential in prostate hyperplasia, and decreases the prostate volume directly. On the other hand, 5ARI have a range of side effects, such as sexual dysfunction. After the discontinuation of 5ARI, prostate regrowth occurs rapidly until it reaches the baseline size. This study examined the effects of 5ARI when used intermittently.

Materials and Methods: Between March 2009 and May 2017, patients who visited one physician's outpatient clinic and were diagnosed with BPH underwent transrectal ultrasonography. The selected patients began to take 5ARI until the prostate size decreased at least $10 \%$ of the baseline (the first medication). After confirming adequate prostate shrinkage, the patients stopped medication until prostate regrowth reached $50 \%$ of the decreased size. After regrowth, they restarted medication for one year (second medication). The prostate size, serum prostate specific antigen (PSA) levels, international prostate symptom score (IPSS) scores, and maximum flow rate (Qmax) in uroflowmetry were collected after the first and second medication and compared using paired t-tests.

Results: Sixty patients with a mean age of 65.1 years were included in the study. The prostate size and serum PSA level increased after the second medication compared to the first, and the prostate reduction and Qmax in uroflowmetry decreased significantly. On the other hand, the symptoms felt by the patients surveyed by the IPSS scores showed no significant difference.

Conclusions: 5ARI appear to be less effective in reducing the prostate volume and improving uroflowmetry after discontinuation.

Keywords: 5-Alpha reductase inhibitors; Prostatic hyperplasia

Copyright $\subset$ 2019, Korean Association of Urogenital Tract Infection and Inflammation. All rights reserved. (c) (1) (\$) This is an open access article distributed under the terms of the Creative Commons Attribution (C) (1) (5) Non-Commercial License (http://creativecommons.org/licenses/by-nc/4.0) which permits unrestricted non-commercial use, distribution, and reproduction in any medium, provided the original work is properly cited.
Received: 6 November, 2019

Revised: 18 November, 2019

Accepted: 19 November, 2019
Correspondence to: Seung Ki Min (iD) https://orcid.org/0000-0002-9638-9668 Department of Urology, National Police Hospital, 123 Songi-ro, Songpa-gu, Seoul 05715, Korea Tel: +82-2-3400-1264, Fax: +82-2-3400-1265 E-mail: drmsk@korea.com

\section{INTRODUCTION}

Benign prostate hyperplasia (BPH) is a disease, in which progressive enlargement of the prostate leads to extrinsic compression of the prostate urethra and impaired voiding [1]. Although BPH is not life threatening, various lower urinary symptoms caused by the disease reduce the quality of life of many senile males [2]. BPH treatments aim to alleviate the urination problems, prevent disease progression, and improve the quality of life [3]. Alpha-adrenergic receptor antagonists ( $\alpha$-blockers) and 5-alpha reductase inhibitors (5ARI) are the most common medical treatments in patients with $\mathrm{BPH}$.

5ARI inhibit the conversion of testosterone to dihydro- 
testosterone, which is essential in prostate hyperplasia, and decrease the prostate size directly, leading to an alleviation of the lower urinary tract symptoms (LUTS) [4]. On the other hand, 5ARI have a range of adverse effects, such as sexual dysfunction and mood disorder, which can reduce the patients' compliance [5]. After discontinuing 5ARI, the prostate volume recovers to the size of pre-medication, and requires re-dosing [6]. Therefore, some urologists repeat the prescription and discontinuation of 5ARI intermittently to reduce the side effects.

A previous study examined the effective factors in prostate recovery after the discontinuation of 5ARI [7]. As an extension, this study examined the patients' clinical course after intermittent 5ARI use.

\section{MATERIALS AND METHODS}

\section{Study Population}

This study was performed retrospectively on BPH patients who visited a doctor's outpatient department between March 2009 and May 2017. After measuring the prostate volumes by transrectal ultrasonography (TRUS), patients with a prostate volume larger than $30 \mathrm{ml}$ were selected as subjects. Patients who underwent BPH-related surgery, such as transurethral resection of the prostate or diagnosed with prostate cancer by a prostate biopsy in the study period, were excluded. The Institutional Review Board of National Police Hospital (no. 11100176-201810-HR-006) approved this study and exempted it from written informed consent.

\section{Study Design}

Fig. 1 shows the course of the study and patient selection. The patients began to take 5ARI (finasteride or dutasteride) after the serum prostate specific antigen (PSA) level was measured. After six months of 5ARI medication, the size of the prostate was re-measured. Patients whose prostate volume was reduced to less than $10 \%$ of the original volume were excluded, and the remaining patients stopped taking 5ARI. After at least one year of discontinuance, a third measurement of the prostate size was performed, and the recovered volume was calculated. 5-ARI medication was restarted when the prostate volume recovered to more than $50 \%$ of its reduced size. After 1-year medication, the final prostate measurement was taken. An international prostate symptom score (IPSS) questionnaire and uroflowmetry were conducted every time TRUS had been performed, and the IPSS scores and maximum flow rate (Qmax) were collected

\section{Statistical Analysis}

After collecting the data, the differences in prostate size, reduced size and rate, PSA scores, IPSS scores, and Qmax between after the first and second medication were analyzed statistically using a paired t-test. SPSS for Windows ver. 12.0 (SPSS Inc., Chicago, IL, USA) was used for all statistical analyses, and two-sided $\mathrm{p}<0.05$ were considered significant.

\section{RESULTS}

Among the patients, 60 patients with a mean age of 65.1 years were included in the study. Table 1 lists the changes in prostate volume, serum PSA levels, IPSS scores, and Qmax measured by uroflowmetry. The mean prostate size, IPSS scores, and Qmax showed improvement after each medication and aggravation after discontinuation. On the other hand, the serum PSA levels showed a different pattern: a decrease from $0.87 \mathrm{ng} / \mathrm{ml}$ at the first medication to 0.57 $\mathrm{ng} / \mathrm{ml}$, but increased from $1.1 \mathrm{ng} / \mathrm{ml}$ at the second medication to $1.37 \mathrm{ng} / \mathrm{ml}$.

The results of the first and second medications were compared statistically and are summarized in Table 2. The mean prostate size increased significantly from $23.5 \mathrm{ml}$ after the first medication to $27.2 \mathrm{ml}$ after the second. The absolute value $(8.8 \mathrm{ml} \rightarrow 7.7 \mathrm{ml})$ and ratio $(27 \% \rightarrow 21 \%)$ of the reduced size compared to the size before medication decreased significantly after the second medication compared to that after the first medication. Qmax $(24.5 \mathrm{ml} / \mathrm{s} \rightarrow 20.6 \mathrm{ml} / \mathrm{s})$ also showed a significant decrease after the second medication compared to that after the first. On the other hand, there were no statistically significant differences in the IPSS scores in both the total and quality of life scores.

\section{DISCUSSION}

During intermittent medication of 5ARI, although both medications show improvements in the prostate volume, Qmax, and IPSS scores, the second medication was less effective in reducing the prostate size and serum PSA level compared to the first medication, which resulted in limited improvement of Qmax by uroflowmetry. On the other hand, there were no significant differences between the symptoms 


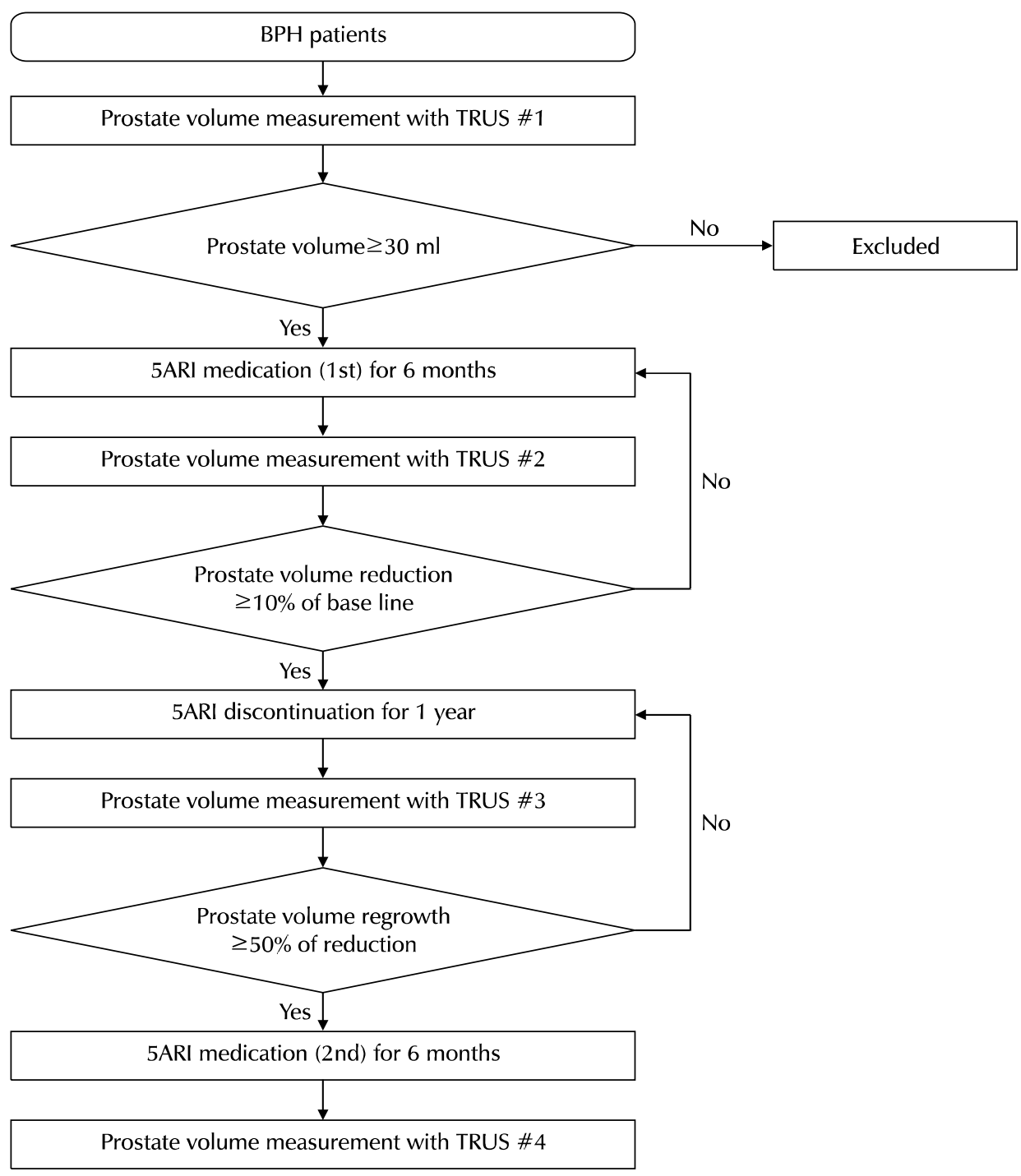

Fig. 1. Flow chart of the study course and patient selection. $\mathrm{BPH}$ : benign prostate hyperplasia, TRUS: transrectal ultrasonography, 5ARI: 5-alpha reductase inhibitors.

Table 1. Changes in the clinical features during the 1st medication, discontinuation, and 2nd medication $(n=60)$

\begin{tabular}{lcccc}
\multicolumn{1}{c}{ Parameters } & Initial & After 1st medication & After discontinuation & After 2nd medication \\
\hline Prostate volume $(\mathrm{ml})$ & $32.3 \pm 4.0$ & $23.5 \pm 3.3$ & $34.9 \pm 5.8$ & $27.2 \pm 6.3$ \\
Serum PSA $(\mathrm{ng} / \mathrm{ml})$ & $0.87 \pm 0.57$ & $0.57 \pm 0.42$ & $1.1 \pm 0.7$ & $1.37 \pm 2.7$ \\
IPSS scores & & & & \\
Total & $11.4 \pm 4.4$ & $9.7 \pm 5.9$ & $10.4 \pm 5.7$ & $9.6 \pm 6.0$ \\
QOL & $3.1 \pm 1.3$ & $2.5 \pm 1.2$ & $3.6 \pm 1.3$ & $2.4 \pm 1.6$ \\
Qmax $(\mathrm{ml} / \mathrm{s})$ & $18.2 \pm 8.2$ & $24.5 \pm 8.6$ & $17.6 \pm 5.4$ & $20.6 \pm 5.9$ \\
\hline
\end{tabular}

Values are presented as mean \pm standard deviation.

PSA: prostate specific antigen, IPSS: international prostate symptom score, QOL: quality of life, Qmax: maximum flow rate.

felt by the patients measured by IPSS after the 1st and 2nd medication. This suggests that the score reflects the patients' satisfaction from the improvement of symptoms after medication, rather than the patient's actual symptoms, but the precise reason may be determined by further long-term research.

$\mathrm{BPH}$ is one of the most common diseases in men aged over 50 years, and its prevalence increases with age [8] Although not life threatening, LUTS caused by BPH, such as hesistancy, poor or intermittent stream, and nocturia, may deteriorate the quality of life. The progressive nature of BPH can be quantified by increases in the LUTS severity according to the IPSS, Qmax, episodes of acute urinary retention, or the need for BPH-related surgery [9]. Two types 
Table 2. Results of statistical analysis between 1 st and 2 nd medication

\begin{tabular}{lccr}
\multicolumn{1}{c}{ Parameters } & $\begin{array}{c}\text { After 1st } \\
\text { medication }\end{array}$ & $\begin{array}{c}\text { After 2nd } \\
\text { medication }\end{array}$ & p-value \\
\hline $\begin{array}{l}\text { Prostate volume }(\mathrm{ml}) \\
\text { Prostate volume reduction }\end{array}$ & $23.5 \pm 3.3$ & $27.2 \pm 6.3$ & $<0.001$ \\
$\quad$ Size $(\mathrm{ml})$ & $8.8 \pm 4.1$ & $7.7 \pm 7.3$ & 0.027 \\
$\quad$ Ratio $(\%)$ & $27 \pm 0.1$ & $21 \pm 0.19$ & $<0.001$ \\
Serum PSA & $0.58 \pm 0.42$ & $1.37 \pm 2.7$ & $<0.001$ \\
IPSS scores & & & \\
$\quad$ Total & $9.7 \pm 5.9$ & $9.6 \pm 6.0$ & 0.473 \\
QOL & $2.5 \pm 1.2$ & $2.4 \pm 1.6$ & 0.388 \\
Qmax $(\mathrm{ml} / \mathrm{s})$ & $24.5 \pm 8.6$ & $20.6 \pm 5.9$ & $<0.001$ \\
\hline
\end{tabular}

Values are presented as mean \pm standard deviation.

PSA: prostate specific antigen, IPSS: international prostate symptom score, QOL: quality of life, Qmax: maximum flow rate.

of treatments are available for patients with BPH: medical treatment and surgical treatment [10]. $\alpha$-blockers, one of first-line medical treatments in $\mathrm{BPH}$, bind to the alpha-1 receptors and relax the smooth-muscle tone in the prostate and bladder neck [11]. $\alpha$-blockers relieve urinary symptoms rapidly and improve the IPSS score and Qmax with few adverse effects [12]. On the other hand, $\alpha$-blockers do not block the progression of prostate enlargement and cannot affect the long-term risk of acute urinary retention or $\mathrm{BPH}-$ related surgery [13].

The other first-line medical treatment is 5ARI. 5ARI blocks the action of 5-alpha reductase, which converts sex-steroid hormone testosterone to dihydrotestosterone, leading to a decrease in the enlarged prostate volume and alleviation of LUTS by BPH. When used as a monotherapy, the speed of symptom control is relatively slower than $\alpha$-blockers. When used in the long term, however, 5-ARI also helps reduce the risk of acute urinary retention and BPH-related surgery [14]. Madersbacher et al. [15] reported that after long-term use of 5ARI, the serum PSA level and prostate volume decreased by approximately $50 \%$ and $20 \%$, respectively. In the present study, after the first medication, the serum PSA level and prostate volume decreased by $33 \%$ and $27 \%$, respectively.

Despite the effects of 5ARI, the range of side effects makes long-term use difficult. Sexual dysfunction, one of the most common side effects, appears in the form of decreased libido, erectile dysfunction, and ejaculation disorder, which especially affects the compliance of relatively younger patients $[16,17]$. In addition, cosmetic changes, such as gynecomastia, and psychiatric side effects, such as anxiety and mood disorders, have been reported $[18,19]$. Because of this, some urologists consider discontinuing 5ARI if the size of the prostate is reduced sufficiently, but the duration of the appropriate treatment is not yet determined.

After discontinuing 5ARI, the prostate size recovered rapidly to its premedication size. Stoner [20] and Kim et al. [6] reported that the prostate regrew to more than $95 \%$ of the baseline after stopping 5ARI for 12 weeks and one year, respectively. In a previous study [7], the mean prostate volume was reduced $28 \%$ during 5 ARI medication but regrew to $97.1 \%$ of the baseline after one year of discontinuation. In this study, the regrowth rate of the prostate reached 108\% after one year of discontinuation. The precise mechanism of this rapid regrowth is unclear, but there are several hypotheses. The hyperplastic changes in $\mathrm{BPH}$ are an androgen-dependent process, and it is possible that the sensitivity to DHT is increased or overly activated because of the prolonged pharmacological inhibition of 5-alpha reductase [21]. Another possible hypothesis is related to the androgen receptors, which become more sensitive or upregulated during treatment with 5ARI, and may be considered as an alternative mechanism [21].

Given that this study is a fragmentary study conducted at a single institution, it had several limitations. First, this study investigated the clinical course of patients who used 5-ARI intermittently. On the other hand, to determine the true significance of intermittent use of the drug, it will be necessary to compare it with patients who continue to use the drug as a control group. Unfortunately, this study could not include those patients owing to a lack of time. Second, two types of 5ARI, finasteride and dutasteride, may have had different effects on the patient's clinical course. The draft of the plan was to separate the two medication groups and compare them statistically. In some cases, however, the two-drugs were used interchangeably, which made it difficult to divide the groups. Because of this, this categorization in this study was excluded. Third, taking $\alpha$-blockers can affect IPSS or uroflowmetry results. Therefore, it is more accurate to unify the use or type of drug in all patients. In that case, however, the number of subjects collected from one institution was so small that the study did not consider whether to take $\alpha$-blockers. Further studies will overcome these limitations.

The strength of this study is that the effects experienced by patients on re-use after discontinuing 5ARI could be observed. Several studies have investigated the clinical 
course of patients, such as prostate regrowth, worsening LUTS, and increasing serum PSA levels, when they stop taking 5ARI, but no study has investigated the clinical course after re-use of 5 ARI.

\section{CONCLUSIONS}

The results showed that after discontinuation, the effects of 5ARI on reducing the prostate size and improving Qmax decreased. On the other hand, there was no difference in the urinary symptoms between the first and second medications. If this is prolonged, it may also influence the effects of 5ARI on decreasing the prevalence of urine retention or BPH-related surgery. Nevertheless, longer-term studies will be needed to demonstrate this. If urologists plan to discontinue 5ARI in BPH patients, they should consider that the drug effects might have a limitation in future re-use, and should consider re-dosing 5ARI before the prostate volume becomes too large. Further studies will help improve the urinary symptoms and minimize the side effects of 5ARI in $\mathrm{BPH}$ patients.

\section{CONFLICT OF INTEREST}

No potential conflict of interest relevant to this article was reported.

\section{ACKNOWLEDGMENTS}

This study was supported by a Korean National Police Hospital Grant.

\section{AUTHOR CONTRIBUTIONS}

K.C. participated in data collection, performed the statistical analysis and wrote the manuscript. B.K. participated in data collection. I.C.C. participated in the the study design and helped to draft the manuscript. S.K.M. participated in the study design and coordination and helped to draft the manuscript. All authors read and approved the final manuscript.

\section{ORCID}

Kwibok Choi, https://orcid.org/0000-0003-3036-4959
Byounghoon Kim, https://orcid.org/0000-0001-9470-8947

In-Chang Cho, https://orcid.org/0000-0001-8906-3478

Seung Ki Min, https://orcid.org/0000-0002-9638-9668

\section{REFERENCES}

1. Boyle P, Roehrborn C, Harkaway R, Logie J, de la Rosette J, Emberton M. 5-Alpha reductase inhibition provides superior benefits to alpha blockade by preventing AUR and BPH-related surgery. Eur Urol 2004;45:620-6; discussion 626-7.

2. Jacobsen SJ, Jacobson DJ, Girman CJ, Roberts RO, Rhodes T, Guess HA, et al. Natural history of prostatism: risk factors for acute urinary retention. J Urol 1997;158:481-7.

3. Oelke M, Bachmann A, Descazeaud A, Emberton M, Gravas S, Michel MC, et al. Guidelines on the management of male lower urinary tract symptoms (LUTS), incl. benign prostatic obstruction (BPO). Arnhem: European Association of Urology; 2013.

4. Marks LS, Andriole GL, Fitzpatrick JM, Schulman CC, Roehrborn CG. The interpretation of serum prostate specific antigen in men receiving 5alpha-reductase inhibitors: a review and clinical recommendations. J Urol 2006;176:868-74.

5. Seftel AD, de la Rosette J, Birt J, Porter V, Zarotsky V, Viktrup L. Coexisting lower urinary tract symptoms and erectile dysfunction: a systematic review of epidemiological data. Int J Clin Pract 2013;67:32-45.

6. Kim W, Jung JH, Kang TW, Song JM, Chung HC. Clinical effects of discontinuing 5-alpha reductase inhibitor in patients with benign prostatic hyperplasia. Korean J Urol 2014;55:52-6.

7. Choi K, Kim B, Cho IC, Min SK. Patient's factors correlated with prostate volume recovery after 5 alpha reductase inhibitor discontinuation. Urogenit Tract Infect 2018;13:79-83.

8. Girman CJ. Population-based studies of the epidemiology of benign prostatic hyperplasia. Br J Urol 1998;82 Suppl 1:34-43.

9. Emberton M, Andriole GL, de la Rosette J, Djavan B, Hoefner K, Vela Navarrete $R$, et al. Benign prostatic hyperplasia: a progressive disease of aging men. Urology 2003;61:267-73.

10. Shin YS, Lee JW, Kim MK, Jeong YB, Park SC. Early dutasteride monotherapy in men with detectable serum prostate-specific antigen levels following radical prostatectomy: a prospective trial. Investig Clin Urol 2017;58:98-102.

11. Kirby RS, Roehrborn C, Boyle P, Bartsch G, Jardin A, Cary MM, et al.; Prospective European Doxazosin and Combination Therapy Study Investigators. Efficacy and tolerability of doxazosin and finasteride, alone or in combination, in treatment of symptomatic benign prostatic hyperplasia: the Prospective European Doxazosin and Combination Therapy (PREDICT) trial. Urology 2003;61:119-26.

12. Djavan B, Marberger M. A meta-analysis on the efficacy and tolerability of alpha1-adrenoceptor antagonists in patients with lower urinary tract symptoms suggestive of benign prostatic obstruction. Eur Urol 1999;36:1-13. 
13. Kaplan SA, Lee JY, Meehan AG, Kusek JW; MTOPS Research Group. Long-term treatment with finasteride improves clinical progression of benign prostatic hyperplasia in men with an enlarged versus a smaller prostate: data from the MTOPS trial. J Urol 2011;185:1369-73.

14. Andersen JT, Nickel JC, Marshall VR, Schulman CC, Boyle P. Finasteride significantly reduces acute urinary retention and need for surgery in patients with symptomatic benign prostatic hyperplasia. Urology 1997;49:839-45.

15. Madersbacher S, Marszalek M, Lackner J, Berger P, Schatzl G. The long-term outcome of medical therapy for BPH. Eur Urol 2007; 51:1522-33.

16. Nickel JC, Fradet Y, Boake RC, Pommerville PJ, Perreault JP, Afridi SK, et al. Efficacy and safety of finasteride therapy for benign prostatic hyperplasia: results of a 2-year randomized controlled trial (the PROSPECT study). PROscar Safety Plus Efficacy Canadian Two Year Study. CMAJ 1996;155:1251-9.

17. Hudson PB, Boake R, Trachtenberg J, Romas NA, Rosenblatt $S$,
Narayan P, et al. Efficacy of finasteride is maintained in patients with benign prostatic hyperplasia treated for 5 years. The North American Finasteride Study Group. Urology 1999;53:690-5.

18. Corona G, Tirabassi G, Santi D, Maseroli E, Gacci M, Dicuio M, et al. Sexual dysfunction in subjects treated with inhibitors of $5 \alpha$-reductase for benign prostatic hyperplasia: a comprehensive review and meta-analysis. Andrology 2017;5:671-8.

19. Trost L, Saitz TR, Hellstrom WJ. Side effects of 5-alpha reductase inhibitors: a comprehensive review. Sex Med Rev 2013;1:24-41.

20. Stoner E. The clinical effects of a 5 alpha-reductase inhibitor, finasteride, on benign prostatic hyperplasia. The Finasteride Study Group. J Urol 1992;147:1298-302.

21. Jeong YB, Kwon KS, Kim SD, Kim HJ. Effect of discontinuation of 5alpha-reductase inhibitors on prostate volume and symptoms in men with BPH: a prospective study. Urology 2009; 73:802-6. 